\title{
Analytical target cascading for optimal configuration of cloud manufacturing services
}

\author{
Yingfeng Zhang ${ }^{\mathrm{a}, \mathrm{b}^{*}}$, Geng Zhang ${ }^{\mathrm{a}}$, Ting $\mathrm{Qu}^{\mathrm{c}}$, Yang Liu ${ }^{\mathrm{c}, \mathrm{de}, * *}$, Ray Y. Zhong ${ }^{\mathrm{f}}$ \\ a Key Laboratory of Contemporary Design and Integrated Manufacturing Technology, Ministry \\ of Education, Northwestern Polytechnical University, Xi'an, 710072, China \\ $b$ Research \& Development Institute in Shenzhen, Northwestern Polytechnical University, \\ Shenzhen, 518057, China \\ c Institute of Physical Internet, Jinan University (Zhuhai Campus), Zhuhai, 519070, China \\ $d$ Department of Management and Engineering, Linköping University, SE-581 83 Linköping, \\ Sweden \\ e Department of Production, University of Vaasa, PL 700, 65101 Vaasa, Finland \\ $f$ Department of Mechanical Engineering, The University of Auckland, Auckland 1142, New \\ Zealand
}

Corresponding Author: zhangyf@nwpu.edu.cn (Y. Zhang), yang.liu@liu.se (Y. Liu)

\begin{abstract}
Combining with advanced technologies (e.g., cloud computing, Internet of Things, and service-oriented technology), cloud manufacturing was proposed and gained wide attention. By managing a huge amount of distributed and idle manufacturing resources to meet various manufacturing requirements, cloud manufacturing provides sustainable means for promoting cleaner production. Manufacturing service configuration plays an important role in implementing cloud manufacturing. Most research adopted central optimization methods to get optimal service configuration results. However, these all-in-one methods with an individual decision model can hardly maintain the autonomous decision rights of different service providers. Consequently, service providers may lose their flexibility to achieve private decision objectives, which is unfavorable for keeping the sustainable competitive advantages of enterprises. In this paper, a decentralized decision mechanism named analytical target cascading is introduced to solve the manufacturing service configuration problem. An analytical target cascading model for the manufacturing service configuration problem is proposed based on the hierarchical structure of cloud manufacturing system. Elements in the proposed model are formulated and solved in a loose coupling and distributed manner. The situation when alternative service providers owned autonomous decision rights to configure their respective upstream manufacturing stages is also considered. A case study is employed to verify the effectiveness of analytical target cascading in solving the manufacturing service configuration problem. It shows that analytical target cascading can not only obtain the same manufacturing service configuration results as central optimization method but also maintain the autonomous decision rights of different service providers.
\end{abstract}

Keywords: cloud manufacturing, manufacturing service configuration, analytical target cascading 


\section{Introduction}

Modern manufacturing mode changed significantly due to the growth of global market competition (Rauch et al., 2016), economic globalization and customer demand diversification (Zhang et al., 2016a). Cloud manufacturing (CMfg) as a new sustainable manufacturing mode has been developed from existing advanced manufacturing modes (e.g., networked manufacturing ( $\mathrm{Li}$ et al., 2015), manufacturing grid (Elkhorchani and Grayaa, 2016)) and enterprise information technologies under the support of Cloud computing (Tao et al., 2014a), Internet of Things (IoT) (Shrouf and Miragliotta, 2015), virtualization and service-oriented technologies.

In CMfg, distributed and idle manufacturing resources are encapsulated into manufacturing services (Xu, 2012). Manufacturing service configuration (MSC) can be defined as a process of manufacturing service composition and optimal selection. As a core part of implementing CMfg, MSC aims to optimize the allocation of manufacturing services for manufacturing tasks. Effective and efficient MSC can not only avoid both over-provisioning and idle resources but also help industries to perform cleaner productions (Sun et al., 2016). MSC has the characteristics of large scale, high heterogeneity, dynamic interconnection and group collaboration (Laili et al., 2012). Besides, real-time information of manufacturing services is acquired and provided for the service configuration by a deeper integration of IoT and CMfg (Qu et al., 2016), which results in the real-time on-demand MSC.

Most research adopted central optimization methods or all-in-one (AIO) methods (e.g., particle swarm optimization, artificial bee colony algorithm) to solve MSC problems. However, some specific challenges still hinder the implementing of sustainable CMfg. Firstly, manufacturing services from different providers probably have different capabilities and provision modes. The service providers may utilize their own decision rights during the service configuration processes. The AIO method with only one decision model can hardly allow service providers to maintain their decision rights. Secondly, a huge amount of manufacturing services from distributed providers are registered in the CMfg platform. Manufacturing tasks are diverse because of various customer demands. If manufacturing tasks with multiple stages are submitted to the CMfg platform, the computation cost would be extremely high. Thirdly, the capabilities of manufacturing services are changing over time along with real-time captured functional status by IoT technologies. It is hard to use AIO methods to include all the service parameters into an individual optimization model.

Analytical target cascading (ATC) is a model-based, multilevel, hierarchical optimization method for system design (Kim et al., 2003a). In the ATC mechanism, a whole system is converted into a hierarchical model with a series of individual elements. Each element has its own decision model, and all elements can be analyzed by a parallel distributed way which greatly reduces the overall computation cost. Due to the promising features of ATC in solving various engineering problems (e.g. automotive vehicle design (Kim et al., 2003b), supply chain configuration (Huang and Qu, 2008)), it is extended for solving MSC problem in this paper.

The primary aim of this research is to investigate how the ATC method can be extended to deal with MSC problem while retaining most of its characteristics of loosely coupled decision making and distributed computing. More specifically, several research questions are focused in this paper:

(1) How to convert the whole manufacturing service configuration problem into a hierarchical ATC model? 
(2) How to maintain the autonomous decision rights of service providers when ATC method is applied to solve the MSC problem?

(3) How to formulate and solve the constructed hierarchical ATC model according to the ATC application procedure?

The rest of this paper is organized as follows. Section 2 reviews the related literature. Section 3 briefly introduces manufacturing services in CMfg platform. An ATC model and key links of MSC problem are described in section 4. Individual elements in the proposed ATC model are formulated in section 5. Section 6 explains ATC solutions for the MSC problem. A case study is given in section 7. Conclusions and future works are drawn in section 8.

\section{Literature review}

Two streams of literature are relevant to this research. They are respectively MSC methods in CMfg and application of ATC in engineering fields.

\subsection{MSC methods in CMfg}

MSC embodies the choice of manufacturing services, their assignments and timing to perform operations while satisfying the manufacturing requirements from customers, processing, and capability constraints. It is a key issue for practical implementation of CMfg. Two research concentrations have been placed upon. The first is about different objectives and constraints for MSC, such as TQCS (time, quality, cost, and service)-based service selection and scheduling (Cao et al., 2016), energy-aware resource service scheduling based on utility evaluation (Cheng et al., 2013), multi-task oriented service composition and optimization (Liu et al., 2013), game theory based real-time scheduling for CMfg (Zhang et al., 2017b), correlation aware service aggregation optimization (Xu et al., 2016). The second is about the algorithms for MSC. For instance, particle swarm optimization for design preference-based and QoS (quality of service)-aware resource service selection (Zheng et al., 2016), chaos control optimal algorithm for QoS based service composition selection (Huang et al., 2014), Pareto group leader algorithm for energy and QoS aware service composition and optimal selection (Xiang et al., 2014), an improved artificial bee colony optimization for QoS and geo-perspective based service composition (Lartigau et al., 2015), case-library method for service composition and optimal selection (Xiang et al., 2016). Although significant progresses were made, several research topics are still needed to be investigated.

- Existing research mainly used AIO methods (such as particle swarm optimization) to consider MSC, whereas the autonomous decision rights of service providers are ignored. However, it is a core element to achieve sustainable competitive advantages (Liu, 2013) through proactive operations (Liu and Liang, 2015). In practice, for example, many related manufacturing service providers often cooperate with each other to complete some complex manufacturing tasks. They are working in a form of alliance. If service peoviders often cooperate together, credibility and reliability of them are higher (Xu et al., 2016). To keep their sustainable competitiveness, service providers prefer to maintain their own decision rights and cooperate with other service providers in the same alliance. A service provider in CMfg platform can be considered as a seller in an E-commerce platform such as eBay. Sellers have rights to decide how to sell their products. Similarly, service providers should have rights to decide how to supply their services.

- The complexity of MSC problem is increased with more and more manufacturing tasks and 
services involved. As the increasing of MSC problem, computation cost will be extremely high since it is prone to solve a NP-hard (non-deterministic polynomial-time hard) problem.

\subsection{Application of ATC method}

Analytical target cascading (ATC) is a method developed for translating system-level design targets to design specifications, which are useful as a coordination method for distributed design optimization of hierarchical, multilevel systems (Tosserams et al., 2008). Based on the illustration in related literaure (Qu et al., 2010a), the working logic of ATC is shown in Fig. 1. In ATC, a system is hierarchically partitioned into several individual elements as the whole complex design problem is converted into a set of sub-problems. Each element represents a sub-problem. Sub-problems are analyzed and solved starting with the elements in the upper level. After analyzing and solving the elements in the upper level, design targets are cascaded to the elements in the lower level. If the lower-level elements cannot achieve the cascaded targets, the upper-level elements are required to adjust the targets; otherwise, cascade the targets until the bottom level elements. When the consistency among all elements is reached, solutions for sub-problems can be obtained and then the final solution for the initial design problem can be found.

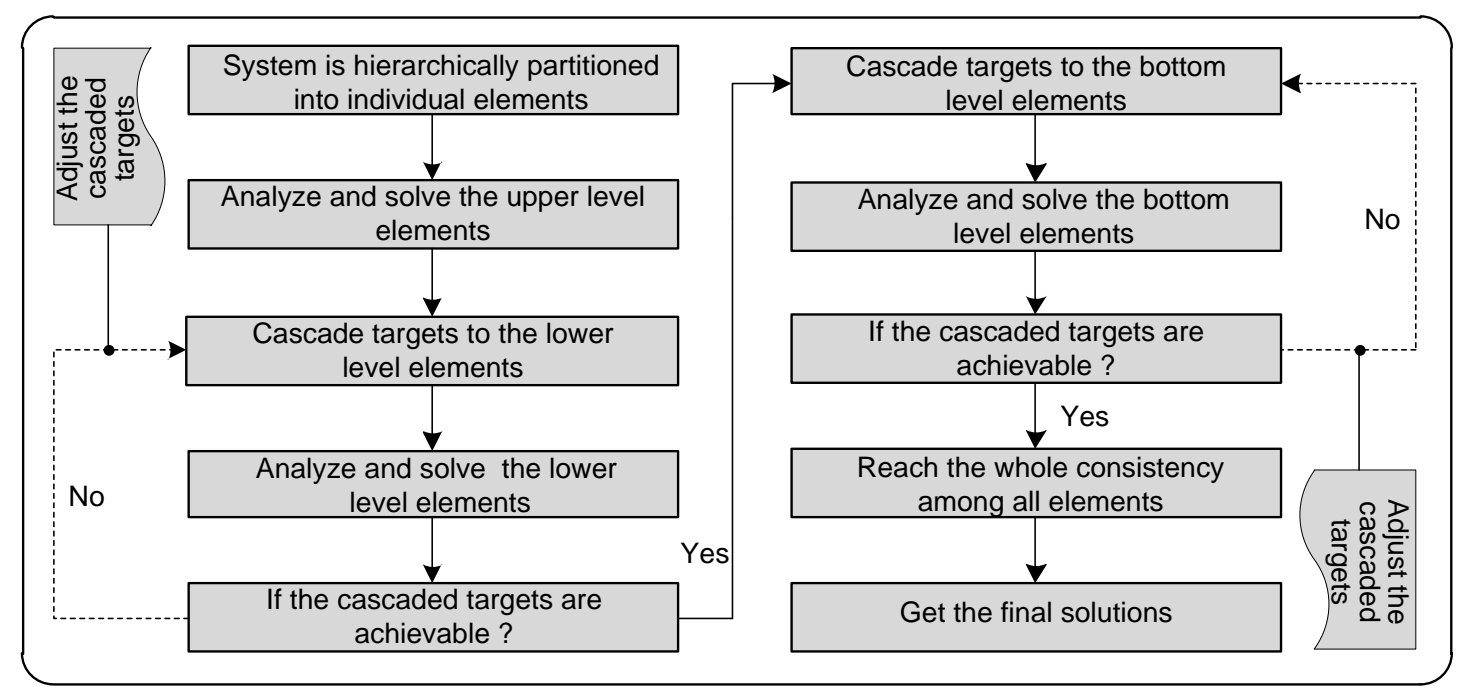

Fig. 1. The working logic of ATC method

ATC method has been widely used in two aspects. One is ATC improvements, such as weighting update method to achieve acceptable consistency deviation in ATC (Michalek and Papalambros, 2005), generalized probabilistic ATC formulation (Liu et al., 2006), extending ATC method to obtain Pareto solutions (Kang et al., 2014b), quadratic exterior penalty function based ATC approach (Jiang et al., 2015) . The other is ATC applications on solving various engineering problems, for instance, automotive chassis design (Kim et al., 2003b), exploring the interrelationships between consumer preference and engineering capabilities (Michalek et al., 2005), formulating and solving the product and process tolerance allocation in multistation compliant assembly ( $\mathrm{Li}$ et al., 2008), developing system to realize decentralized supply chain configuration over supply chain grid (Qu et al., 2010b), optimal design of commercial vehicle system (Kang et al., 2014a). Some observations can be drawn as follows:

- In ATC method, individual elements can have their own decision models. Therefore, their autonomous decision rights can be maintained during the whole optimization process. 
- ATC method adopts a distributed optimization and parallel computing way to achieve optimal solutions, which can improve overall optimization efficiency. Therefore, the computing cost won't be greatly increased when the complexity of problems becomes higher and higher.

To tackle the challenges in MSC by integrating ATC method, this paper takes the example of manufacturing services in CMfg to examine the effectiveness and efficiency from a real-life case.

\section{Manufacturing services in CMfg}

As shown in Fig.2, there are three kinds of users in a CMfg platform. They are cloud operator, cloud service provider, and cloud customer (Buckholtz et al., 2015). Cloud operator is the owner of CMfg platform. It is responsible for setting up operation mechanisms and rules (e.g., technical process and business strategy) and ensuring the communication between service provider and cloud customer. Cloud operator benefits from creating value for cloud customer and service provider. Service provider offers manufacturing service for specific manufacturing demands. Cloud customer is the receipt of manufacturing services. To achieve the optimal configuration of cloud manufacturing service, necessary information is shared among them. The necessary information can be divided into two parts, namely requirement information and capability information. Requirement information is about manufacturing requirements for a demanded product, such as product features, due time, maximum cost, etc. It is submitted to the CMfg platform by the cloud customer. Capability information indicates manufacturing capabilities of service, such as processing method, processing precision, service cost, service time, etc. It is submitted to the CMfg platform by the service provider. After receiving requirement information from cloud customer, the CMfg platform will search candidate services to meet the manufacturing requirements based on submitted capability information.

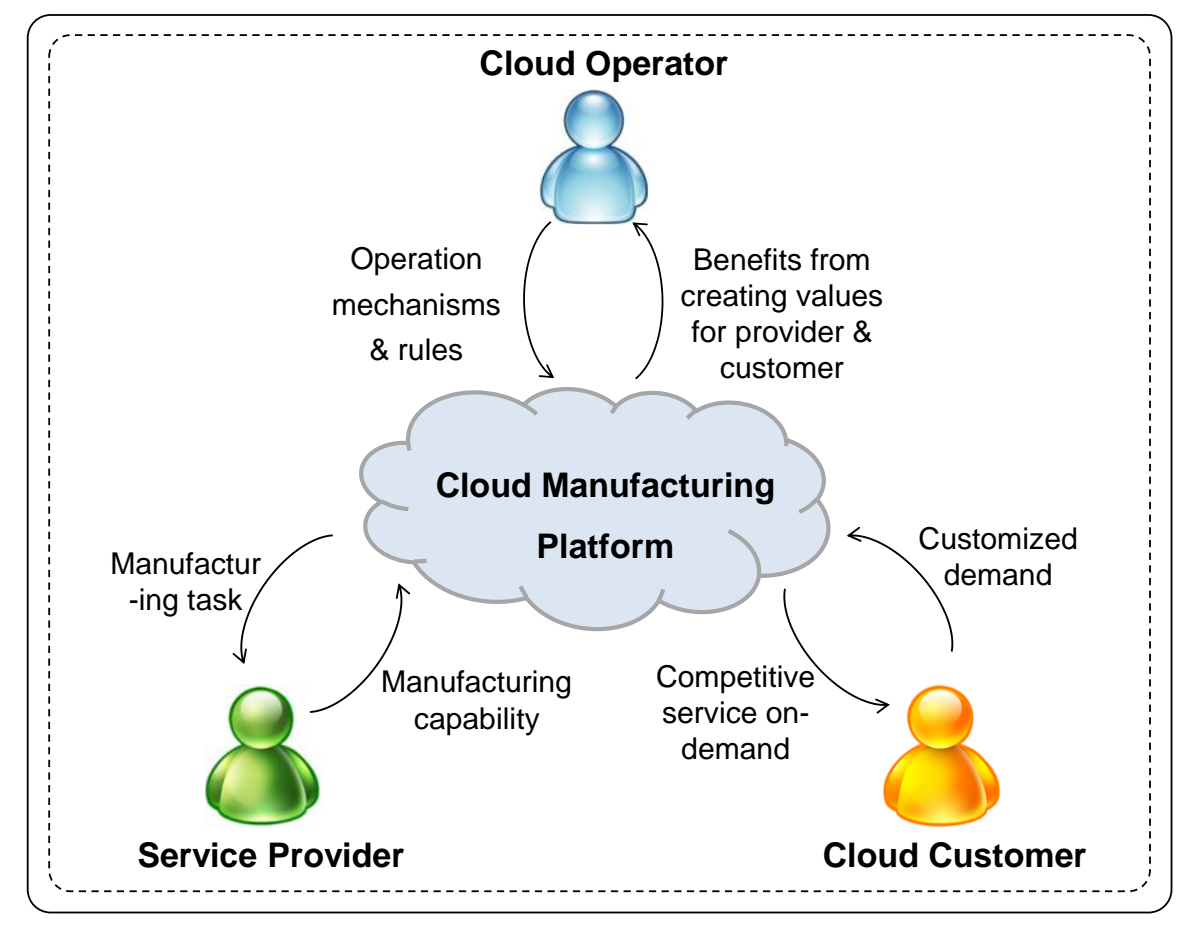

Fig. 2. Users in Cloud manufacturing (Adopted from (Ren et al., 2013)) 
Manufacturing services in CMfg are the results of virtualization and encapsulation of various manufacturing resources with related manufacturing capabilities. Manufacturing resources primarily refer to the resources involved in each production action during the entire life cycle of a product (Tao et al., 2014b). The manufacturing resources in this paper mainly refer to the resources on shop-floors, such as manufacturing systems, manufacturing cells and machines whose detailed information is given in section 4. Manufacturing capabilities refer to the typical abilities of production resources.

Fig. 3 shows a working logic of cloud manufacturing service. It contains three parts. The first part is about the encapsulation of manufacturing resources and capabilities. Manufacturing resources with related capabilities are perceived through advanced technologies, such as IoT (Zhang et al., 2016b), network technology and sensors (Zhang et al., 2017a). Then the perceived manufacturing resources and capabilities are encapsulated into manufacturing services under the support of service-oriented technology. The second part is publishing of manufacturing services. Manufacturing services are published to CMfg platform so that they can be allocated to manufacturing tasks. Technologies such as semantic web service and UDDI (Universal Description, Discovery, and Integration) can make manufacturing services access to CMfg platform. These two parts are explained in detailed in the previous research work (Zhang et al., 2015). The third part is about manufacturing services matching with manufacturing task. The published manufacturing services are managed in a pool. After MSC, an enterprise can obtain a manufacturing task from the common CMfg platform. Then the enterprise allocates right services (e.g. machines, manufacturing cells) to accomplish the manufacturing task.

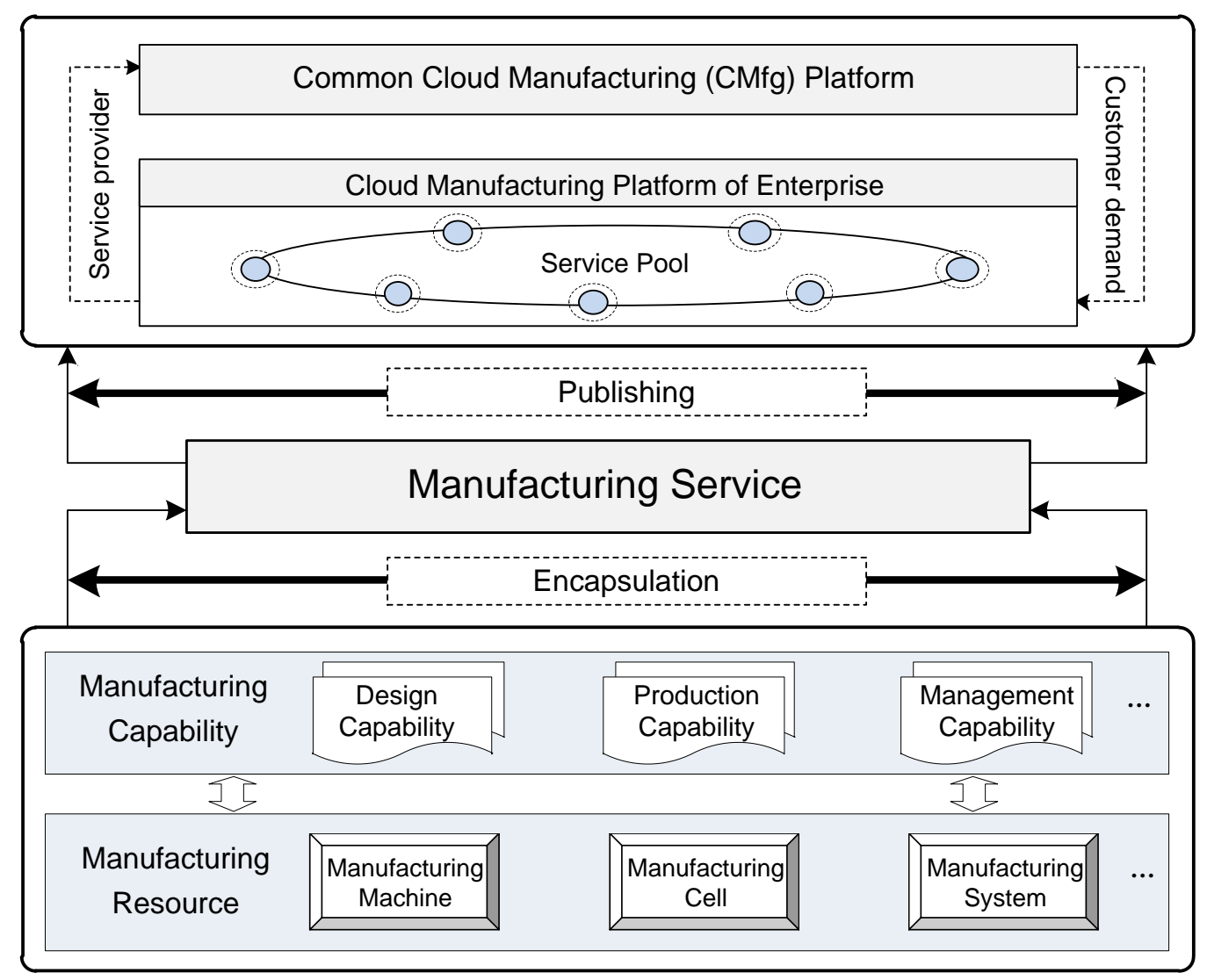

Fig. 3. Working logic of cloud manufacturing service 


\section{ATC model and key links of MSC problem}

Five steps are involved in the application of ATC. The first step is to partition a system into a hierarchical model. The second step is to identify the key links between individual elements in the hierarchical model. The third step is to formulate local problems for each element. ATC solutions and results analysis are respectively the fourth step and fifth step. These five steps are generally followed in applying ATC to solve MSC problem. The first two steps are described in this section and the rest in the following sections.

\subsection{ATC model of MSC problem}

The first step is to create a hierarchical ATC model of MSC problem. Before creating the ATC model, two related points should be mentioned here. Firstly, a manufacturing task submitted to the CMfg platform generally contains many different manufacturing stages. Each manufacturing stage may be composed of some sub-stages which follow a hierarchical structure. Secondly, manufacturing services registered in the CMfg platform are organized in a way of management pyramid which also follows a hierarchical structure. In CMfg, ATC model of MSC problem is created based on the hierarchical structures of manufacturing tasks and services.

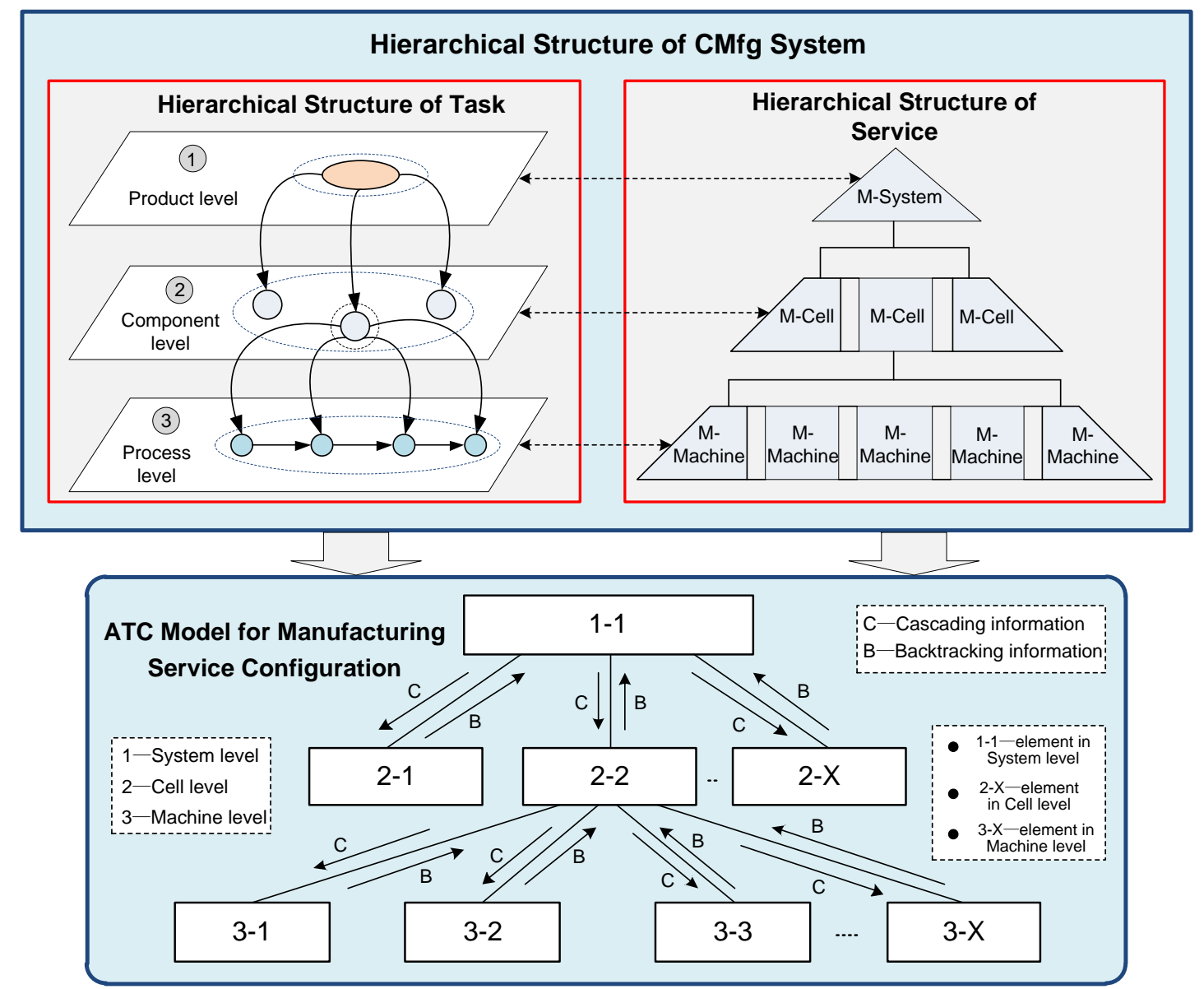

Fig. 4. ATC model of manufacturing service configuration

The upper part of Fig. 4 shows the hierarchical structure of CMfg system. It consists of manufacturing tasks and services in a form of hierarchy. The hierarchical structure of a manufacturing task is presented as one network of manufacturing nodes which can represent 
different manufacturing stages. The network is composed of three different levels, namely product level, component level and process level. Each level has some nodes to represent different manufacturing demands. The nodes in product level show the product requests from clients. The nodes in component level indicate necessary components to form the required product. The nodes in process level are series of processes. Manufacturing resources are encapsulated into services to meet the demands of manufacturing nodes. There are three kinds of manufacturing resources included in the hierarchical structure, namely manufacturing system, manufacturing cell and machine. Generally, a manufacturing system has many manufacturing cells each of which is logically and physically made up of several machines. The manufacturing system is responsible for the demands of product node. Manufacturing cells are employed to manage the component nodes. Machines are used to deal with the process nodes.

The lower part of Fig. 4 shows the ATC model of MSC problem. The manufacturing service network for a manufacturing task is partitioned into a three-level hierarchy composed of ATC elements which are system level, cell level and machine level. Each element in the ATC model denotes a manufacturing node in the hierarchical structure of manufacturing task aiming to find suitable manufacturing service for the corresponding manufacturing node. An assumption that the same manufacturing service could not be provided for different manufacturing nodes is made in this ATC model. In other words, there is no common manufacturing node in the ATC model. Therefore, the partitioned model has a strictly hierarchical structure. The system level element, 1-1, has many child elements (e.g. 2-1 and 2-2) in the cell level. Elements in cell level also have many child elements in the machine level. Element 2-2, for instance, has the child elements 3-1, 3-2, etc. Cascading information and backtracking information are shared between parent and child elements.

For simplicity of understanding but without losing generality of principle, some assumptions are made: 1) Candidate services for each manufacturing stage are selected based on their manufacturing capabilities. 2) All selected candidate services can meet both qualitative and legal requirements for their corresponding manufacturing stages. That means service providers can qualify from qualitative aspects and legal requirements for specific parts in the model. 3) Candidate services have the same value of logistics properties such as logistics distance between related services, transport cost, transport efficiency, etc. Therefore, the influence of logistics on MSC is not considered in this paper.

\subsection{Key links between individual ATC elements}

The second step is to identify the key links of individual elements in the ATC model. For simplicity of understanding but without losing generality of principle, a case ATC model of MSC problem is presented in Fig. 5. 


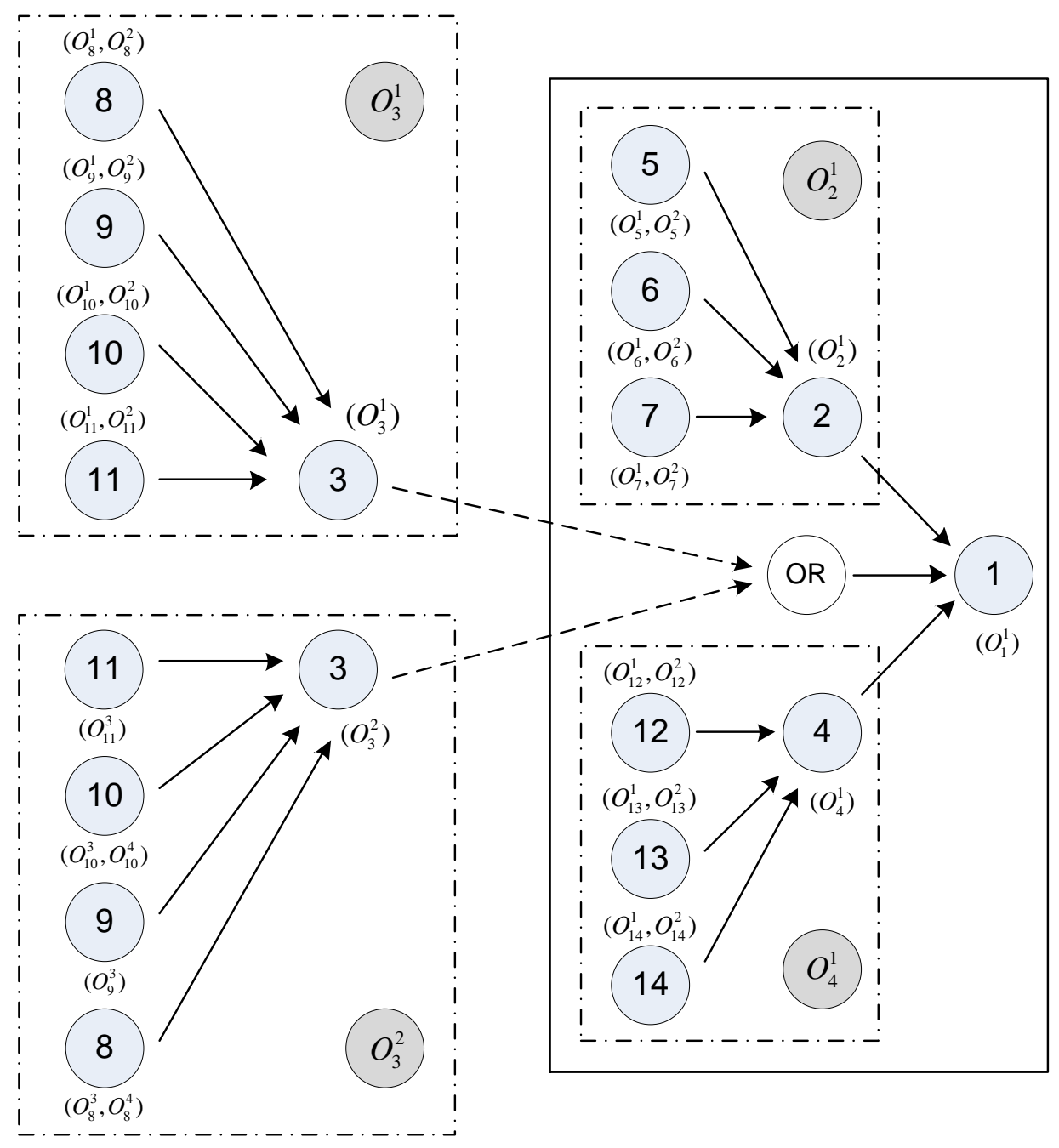

Fig. 5. A case of ATC model

Notations used in this paper are introduced as follows.

$O_{i}^{j}: j^{\text {th }}$ manufacturing service for ATC element $i$ or manufacturing node (stage) $i$;

$O_{i}:$ Candidate services set for element $i, O_{i}=\left\{O_{i}^{1}, O_{i}^{2}, \ldots, O_{i}^{j}, \ldots\right\}$

$v(i)$ : Child element set of element $i$

$T T_{i}$ : Total manufacturing time for completing element $i$

$T C_{i}$ : Total manufacturing cost for completing element $i$

$T E_{i}$ : Total energy consumption for completing element $i$

$T_{i}$ : Local manufacturing time for completing element $i$

$C_{i}$ : Local manufacturing cost for completing element $i$ 
$E_{i}$ : Local energy consumption for completing element $i$;

$t_{o_{i}^{j}}$ : Manufacturing time of service option $j$ completing element $i$;

$c_{O_{i}^{j}}$ : Manufacturing cost of service option $j$ completing element $i$ in system level or machine level; maintenance cost per unit time of service option $\mathrm{j}$ completing element $i$ in cell level;

$e_{O_{i}^{j}}$ : Energy consumption per unit time of service option $j$ completing element $i$ in system level or machine level; energy consumption per unit time of service option $j$ completing element $i$ in cell level;

$S_{O_{i}^{j}}$ : Selecting parameter. When service $O_{i}^{j}$ is selected to complete element $i, s_{O_{i}^{j}}=1$; otherwise, $s_{O_{i}^{j}}=0$.

As shown in Fig. 5, service options are given in the bracket near the corresponding elements. Services with grey circles like $O_{2}^{1}, O_{3}^{1}, O_{3}^{2}$ and $O_{4}^{1}$ utilize independent decision rights to configure their respective upstream elements which are in same dotted blocks. According to different manufacturing scopes, elements in the presented ATC model are categorized into four sets. The first set (system level) includes the most downstream element 1 , which represents the product demand and assembly stage. The second set (cell level) includes elements 2, 3 and 4 which perform the production of required components. The third set (machine level set) includes all the most upstream elements which represent the necessary processes of each component, Elements 5, 6, 7, 8, 9, 10, 11, 12,13 , and 14 belong to this set. Especially, two alternative manufacturing cells, $O_{3}^{1}$ and $O_{3}^{2}$ are available in element 3 for selection. They compete against each other and only one of them can be selected. For this circumstance, an "OR" element is introduced to formally represent the alternative elements. It was successfully used for the optimal configuration of supply chain with alternative autonomous suppliers by Huang et al (2008). In this paper, the "OR" element is used to accomplish the selection among alternative options $O_{3}^{1}$ and $O_{3}^{2}$. It is considered as the fourth set. The working logic of "OR" element is explained in section 5.

In this paper, the objective is to minimize the sum of weighted total manufacturing time, total manufacturing cost and total energy consumption. The mathematical model of this problem can be stated as follows:

Objective function $\quad \min w_{1}^{T} \cdot T T_{1}+w_{1}^{C} \cdot T C_{1}+w_{1}^{E} \cdot T E_{1}$

Subject to $T T_{i}=\left\{\begin{array}{l}T_{i}+\max \left\{T T_{k} \mid k \in v(i)\right\}, \text { for system level set } \\ T_{i}+\sum_{k \in v(i)} T T_{k}, \text { otherwise }\end{array}\right.$

$$
T C_{i}=C_{i}+\sum_{k \in v(i)} T C_{k}
$$




$$
\begin{aligned}
& T E_{i}=E_{i}+\sum_{k \in v(i)} T E_{k} \\
& T_{i}=\sum s_{O_{i}^{j}} \cdot t_{O_{i}^{j}} \\
& C_{i}=\left\{\begin{array}{l}
\sum_{O_{i}^{j} \in O_{i}} s_{O_{i}^{j}} \cdot c_{O_{i}^{j}} \cdot T T_{i}, \text { for the cell level set } \\
\sum_{O_{i}^{j} \in O_{i}} s_{O_{i}^{j}} \cdot c_{O_{i}^{j}}, \text { otherwise }
\end{array}\right. \\
& E_{i}=\left\{\begin{array}{l}
\sum_{O_{i}^{j} \in O_{i}} s_{O_{i}^{j}} \cdot e_{O_{i}^{j}} \cdot T T_{i}, \text { for the cell level set } \\
\sum_{O_{i}^{j} \in O_{i}} s_{O_{i}^{j}} \cdot e_{O_{i}^{j}} \cdot t_{O_{i}^{j}}, \text { otherwise }
\end{array}\right. \\
& \sum_{O_{i}^{j} \in O_{i}} S_{O_{i}^{j}}=1
\end{aligned}
$$

Three parts are included in the objective function. They are the total manufacturing time, manufacturing cost and energy consumption respectively. Energy consumption can be a subset of manufacturing cost to some extent. For example, the electric energy consumption during production process can be evaluated. Reducing energy consumption plays a vital role in achieving sustainable and cleaner CMfg. Due to the increasing price of energy in manufacturing, energy consumption is set as an individual cost in this paper. Equations (2)-(4) are used to get the total manufacturing time, manufacturing cost and energy consumption for completing each element (or manufacturing stage). Equations (5)-(7) are employed to calculate local manufacturing time, manufacturing cost and energy consumption for completing each element. Equation (8) ensures that only one manufacturing service is selected to complete element $\mathrm{i}$.

Key links are the variables shared by elements in the ATC model. It includes two kinds of variables, responses shared by parent and child elements, and linking variables shared by child elements from a same parent element. In this case, key links mainly refer to response variables. The identification of responses starts from the most downstream element 1 . Considering the manufacturing requirements, the total manufacturing cost $T C_{1}$, manufacturing time $T T_{1}$ and energy consumption $T E_{1}$ should be minimized. Nine variables are used in calculating $T C_{1}, T T_{1}$ and $T E_{1}$. They are responses from elements 2,4 , and "OR". The nine variables are respectively $T C_{2}, T T_{2}, T E_{2}, T C_{4}, T T_{4}, T E_{4}, T C_{O R}, T T_{O R}$, and $T E_{O R}$. They are the so-called key links which are shared between element 1 and its child elements (e.g. $T C_{2}, T T_{2}$ and $T E_{2}$ are the key links shared between element 1 and 2). Similarly, the analysis models for the nine variables also require responses from upstream elements. This process will be propagated until responses of the most upstream elements are identified. 


\section{ATC formulations for individual elements}

The third step is to formulate individual elements in the model. Three major parts are included in an ATC formulation. They are objective functions, analysis models, and constraints. The objective function aims to minimize the deviations of responses and linking variables between related ATC elements. The analysis models are used to generate the responses of concerned elements. The constraints are employed to keep the consistency between related elements. Since the similarity of formulations for elements in the same set, only one element in each set is chosen to illustrate. In this paper, objective functions are shown by "Minimize" items, analysis models are shown by "Where" items and constraints are shown by "Subject to" items.

\subsection{ATC formulations for system level set}

Only element 1 is included in the system level set. The optimization of system level set aims to minimize the deviation between element responses and manufacturing targets, which subjects to the manufacturing constraints and tolerance constraints. The formulation for element 1 is presented as follows,

$$
\begin{aligned}
& \text { Minimize }\left\|\left(w_{1}^{T} \cdot\left(T T_{1}-t^{T}\right), w_{1}^{C} \cdot\left(T C_{1}-t^{C}\right), w_{1}^{E} \cdot\left(T E_{1}-t^{E}\right)\right)\right\|_{2}^{2}+\sum_{i=1}^{7} \varepsilon_{1 . i} \\
& \qquad \begin{array}{c}
T T_{1}=T_{1 \cdot \max }+T_{1} \\
T C_{1}=T C_{2}+T C_{O R}+T C_{4}+C_{1} \\
\qquad E_{1}=T E_{2}+T E_{O R}+T E_{4}+E_{1} \\
T_{1 \cdot \max }=\max \left\{T T_{2}, T T_{O R}, T T_{4}\right\} \\
T_{1}=t_{O_{1}^{1}}, C_{1}=c_{O_{1}^{1}}, E_{1}=e_{O_{1}^{1}} \cdot t_{O_{1}^{1}}
\end{array}
\end{aligned}
$$

Subject to $\quad\left|T_{1 \cdot \max }-\max \left\{T T_{2}^{2}, T T_{O R}^{O R}, T T_{4}^{4}\right\}\right| \leq \varepsilon_{1.1}$

$$
\begin{gathered}
\left\|T C_{2}-T C_{2}^{2}\right\|_{2}^{2} \leq \varepsilon_{1.2},\left\|T C_{O R}-T C_{O R}^{O R}\right\|_{2}^{2} \leq \varepsilon_{1.3},\left\|T C_{4}-T C_{4}^{4}\right\|_{2}^{2} \leq \varepsilon_{1.4} \\
\left\|T E_{2}-T E_{2}^{2}\right\|_{2}^{2} \leq \varepsilon_{1.5},\left\|T E_{O R}-T E_{O R}^{O R}\right\|_{2}^{2} \leq \varepsilon_{1.6},\left\|T E_{4}-T E_{4}^{4}\right\|_{2}^{2} \leq \varepsilon_{1.7} \\
T T_{2}, T T_{O R}, T T_{4}, T C_{2}, T C_{O R}, T C_{4}, T E_{2}, T E_{O R}, T E_{4} \geq 0
\end{gathered}
$$

- Objective function: $t^{T}, t^{C}, t^{E}$ are manufacturing targets for total time, total cost, and total energy consumption, which are normally set to zero to get the minimal manufacturing time, cost, and energy consumption. $T T_{1}, T C_{1}$ and $T E_{1}$ are responses from the analysis models of element 1. $w_{1}^{T}, w_{1}^{C}$ and $w_{1}^{E}$ are weighting coefficients for the deviation terms, which indicate the 
preferences of customers. $\varepsilon_{1 . i}$ is the tolerance variable for the response from upstream element. It's used to keep consistency between related elements. The square of $l_{2}$-norm, $\|\cdot\|_{2}^{2}$, is used to measure the deviations between targets and responses.

- Analysis model: $T T_{1}$ is the summation of $T_{1}$ (manufacturing time of element 1 ) and $T_{1 \cdot \max }$ (maximum manufacturing time of its upstream elements). Similarly, $T C_{1}$ is the summation of manufacturing cost of element 1 and its upstream elements. $T E_{1}$ is the summation of energy consumption of element 1 and its upstream elements. $T_{1}$ is equal to $t_{O_{1}^{1}}$ (manufacturing time of $\left.O_{1}^{1}\right) . C_{1}$ is equal to $c_{O_{1}^{1}}$ (manufacturing cost of $O_{1}^{1}$ ). $E_{1}$ is equal to the product of $t_{O_{1}^{1}}$ and $e_{O_{1}^{1}}$ (energy consumption per unit manufacturing time of $O_{1}^{1}$ ).

- Constraints: “ $R_{a}^{b}$ ” (i.e. $R \in\{T T, T C, T E\}$ ) represents the target (or response) of element $a$ which is cascaded (or backtracked) from element $b$. For example, $T T_{2}^{2}$ means the backtracked total manufacturing time of element 2. Constraints (15)-(17) are used to limit the variations of responses from upstream elements within corresponding tolerance variables, e.g. constraint (15) indicates that the variations between $T_{1 . \max }$ and its response from upstream elements should be limited within the tolerance variable $\varepsilon_{1.1}$. The last constraint requires that all the responses about manufacturing cost, time and energy consumption cascaded to upstream elements, should be positive values.

\subsection{ATC formulations for cell level set}

There are three elements included in the cell level set, namely element 2, element 3 and element 4. The optimization of cell level set aims to minimize the deviations between element responses and their targets from downstream elements, which subject to the manufacturing constraints and the tolerance constraints that coordinate upstream elements. Take element 2 as an illustration, its formulation is presented as follows:

$$
\begin{aligned}
& \text { Minimize }\left\|\left(w_{2}^{T} \cdot\left(T T_{2}-T T_{2}^{1}\right), w_{2}^{C} \cdot\left(T C_{2}-T C_{2}^{1}\right), w_{2}^{E} \cdot\left(T E_{2}-T E_{2}^{1}\right)\right)\right\|_{2}^{2}+\sum_{i=1}^{9} \varepsilon_{2 . i} \\
& \qquad \begin{array}{l}
T T_{2}=T T_{5}+T T_{6}+T T_{7}+T_{2} \\
T C_{2}=T C_{5}+T C_{6}+T C_{7}+C_{2} \\
T E_{2}=T E_{5}+T E_{6}+T E_{7}+E_{2}
\end{array}
\end{aligned}
$$




$$
T_{2}=t_{O_{2}^{1}}, C_{2}=c_{O_{2}^{1}} \cdot T T_{2}, E_{2}=e_{O_{2}^{1}} \cdot T T_{2}
$$

Subject to

$$
\begin{gathered}
\left\|T T_{k}-T T_{k}^{k}\right\|_{2}^{2} \leq \varepsilon_{2 .(k-4)} \\
\left\|T C_{k}-T C_{k}^{k}\right\|_{2}^{2} \leq \varepsilon_{2 .(k-1)} \\
\left\|T E_{k}-T E_{k}^{k}\right\|_{2}^{2} \leq \varepsilon_{2 .(k+2)} \\
T T_{k}, T C_{k}, T E_{k} \geq 0 \quad k=5,6,7
\end{gathered}
$$

- Objective function: Three deviation terms and nine tolerance variables are included in the objective function of element 2. $T T_{2}, T C_{2}$ and $T E_{2}$ are the responses of element 2. $T T_{2}^{1}, T C_{2}^{1}$ and $T E_{2}^{1}$ from the optimization results of element 1 are the targets for $T T_{2}, T C_{2}$ and $T E_{2} . w_{2}^{T}$, $w_{2}^{C}$ and $w_{2}^{E}$ are weighting coefficients for the deviation terms. Nine tolerance variables are used to coordinate responses from the upstream elements $(5,6$, and 7$)$.

- Analysis model: To get the responses, necessary analysis models are presented in the formulation. $T T_{2}$ is denoted by the summation of manufacturing time of element 2 and its upstream elements. Similarly, $T C_{2}$ is denoted by the summation of manufacturing cost of element 2 and its upstream elements. $T E_{2}$ is denoted by the summation of energy consumption of element 1 and its upstream elements. $T_{2}$ is equal to $t_{O_{2}^{1}}$ (manufacturing time of $O_{2}^{1}$ ). The manufacturing cost of element 2 refers to the maintenance cost. $C_{2}$ is equal to the product of $T T_{2}$ and $c_{O_{2}^{1}}$ (maintenance cost per unit time of $O_{2}^{1}$ ). $E_{2}$ is equal to the product of $T T_{2}$ and $e_{O_{2}^{1}}$ (energy consumption per unit time of $\mathrm{O}_{2}^{1}$ ).

- Constraints: As explained in 5.1, the constraints here are also used to limit the variations of responses from upstream elements within corresponding tolerance variables. In addition, the last constraint aims to meet the manufacturing requirement that the responses about manufacturing cost, time and energy consumption of upstream elements should be positive.

\subsection{ATC formulation for machine level set}

As discussed in section 4, there are ten elements in the machine level set. They are the most upstream elements in this ATC model. The local optimization of machine level set aims to minimize the deviations between element responses and the targets from the cell level set. Take element 5 as an illustration, its formulation is presented as follows:

$$
\text { Minimize } \quad\left\|w_{5}^{T} \cdot\left(T T_{5}-T T_{5}^{2}\right), w_{5}^{C} \cdot\left(T C_{5}-T C_{5}^{2}\right), w_{5}^{E} \cdot\left(T E_{5}-T E_{5}^{2}\right)\right\|_{2}^{2}
$$


Where

$$
\begin{gathered}
T T_{5}=T_{5} \\
T C_{5}=C_{5} \\
T E_{5}=E_{5} \\
T_{5}=\sum_{j=1}^{2} s_{O_{5}^{j}} \cdot t_{O_{5}^{j}} \\
C_{5}=\sum_{j=1}^{2} s_{O_{5}^{j}} \cdot c_{O_{5}^{j}} \\
E_{5}=\sum_{j=1}^{2} s_{O_{5}^{j}} \cdot e_{O_{5}^{j}} \cdot t_{O_{5}^{j}} \\
=\left\{\begin{array}{l}
1 \text { if } O_{5}^{j} \text { is selected } \\
0 \text { otherwise }
\end{array} \text { and } \sum_{j=1}^{2} s_{O_{5}^{j}}=1\right.
\end{gathered}
$$

- Objective function: Element 5 has no upstream elements, thus there are no tolerance variables included in the objective function. $T T_{5}, T C_{5}$ and $T E_{5}$ are the responses of element 5. $T T_{5}^{2}$, $T C_{5}^{2}$ and $T E_{5}^{2}$ are the targets for responses, which come from element $2 . w_{5}^{T}, w_{5}^{C}$ and $w_{5}^{E}$ are weighting coefficients for the deviation terms.

- Analysis model: Because element 5 has no upstream elements, so the response $T T_{5}$ only contains the local manufacturing time. $T C_{5}$ only contains the local manufacturing cost and $T E_{5}$ only contains the local energy consumption.

- Constraints: Equations (32)-(34) are used to get the local manufacturing time, manufacturing cost and energy consumption for completing element 5. Constraint (35) is used to make sure that only one alternative service will be selected to accomplish the process which element 5 indicates.

\subsection{ATC formulations for OR element}

As mentioned previously, OR element is a special existence in the ATC model. It is used to select the best alternative for element 3 during maintaining the decision right of manufacturing cells, i.e. $O_{3}^{1}$ and $O_{3}^{2}$. It also has a unique working logic of its own. After receiving cascaded targets from element 1, OR element cascades the targets to the alternative elements $O_{3}^{1}$ and $O_{3}^{2}$. Then, the alternative elements execute their local optimization respectively and return responses to OR element. After all the responses are returned, an evaluation function is used to calculate the deviations between the responses and targets. The alternative with smallest deviations is regarded as the best service for element 3 in this cycle. The responses of the best alternative are backtracked to 
element 1 . In brief, the optimization of OR element aims to find the alternative which has the minimal deviations between its responses and the cascaded targets. The formulation for OR element is presented as follows:

$$
\begin{aligned}
& \text { Minimize } \|\left(w_{O R}^{T} \cdot\left(T T_{O R}-T_{O R}^{1}\right), w_{O R}^{C} \cdot\left(T C_{O R}-T C_{O R}^{1}\right), w_{O R}^{E} \cdot\left(T E_{O R}-T E_{O R}^{1}\right) \|_{2}^{2}\right. \\
& \text { Where } \\
& \qquad T_{O R}=\sum_{j=1}^{2} s_{O_{3}^{j}} \cdot T T_{O_{3}^{j}}=\sum_{j=1}^{2} s_{O_{3}^{j}} \cdot T C_{O_{3}^{j}} \\
& T E_{O R}=\sum_{j=1}^{2} s_{O_{3}^{j}} \cdot T E_{O_{3}^{j}} \\
& \text { Subject to } \quad s_{O_{3}^{j}}=\left\{\begin{array}{l}
1 \text { if } O_{3}^{j} \text { is selected and } \sum_{j=1}^{2} s_{O_{3}^{j}}=1 \\
0 \text { otherwise }
\end{array}\right.
\end{aligned}
$$

- Objective function: Three deviation terms are included in the objective function. $T T_{O R}, T C_{O R}$ and $T E_{O R}$ are responses from the analysis models of OR element. $T T_{O R}$ is defined as the total manufacturing time of OR element. $T C_{O R}$ denotes the total manufacturing cost of OR element. $T E_{O R}$ represents the total energy consumption of OR element. $T T_{O R}^{1}, T C_{O R}^{1}$ and $T E_{O R}^{1}$ are respectively the targets for $T T_{O R}, T C_{O R}$ and $T E_{O R} \cdot w_{O R}^{T}, w_{O R}^{C}$ and $w_{O R}^{E}$ are weighting coefficients for the deviation terms. The object function of OR element can also be considered as the evaluation function to choose the best alternative from $O_{3}^{1}$ and $O_{3}^{2}$.

- Analysis model: The local optimization process of OR element is a case of integer programming. Therefore, a standard method can be used to present the responses of OR element. $O_{3}^{j}$ denotes the $j$ th $(1 \leq j \leq 2$ and $j \in N)$ alternative. $T T_{O_{3}^{j}}, T C_{O_{3}^{j}}$ and $T E_{O_{3}^{j}}$ are the responses backtracked from the $j$ th alternative. $s_{O_{3}^{j}}$ is a variable to decide whether the $j$ th alternative is selected. The summations of $s_{O_{3}^{j}} \cdot R_{O_{3}^{j}}\left(R_{O_{3}^{j}} \in\left\{T T_{O_{3}^{j}}, T C_{O_{3}^{j}}, T E_{O_{3}^{j}}\right\}\right)$ from all alternatives are responses of OR element.

- Constraints: The constraints are used to make sure that only one alternative will be selected in the optimization process. When the $j$ th alternative is selected, $s_{O_{3}^{j}}=1$; otherwise, $s_{O_{3}^{j}}=0$.

Because only one alternative will be selected, $\sum_{j=1}^{2} s_{O_{3}^{j}}$ should be 1. 


\section{ATC solutions for MSC problem}

The fourth step is to solve the formulated ATC problems by adopting right solutions. The ATC solutions can be summarized as two aspects. The first is about the convergence strategy for the whole hierarchical ATC model and the second is about optimization method for each individual element.

\subsection{Convergence strategy}

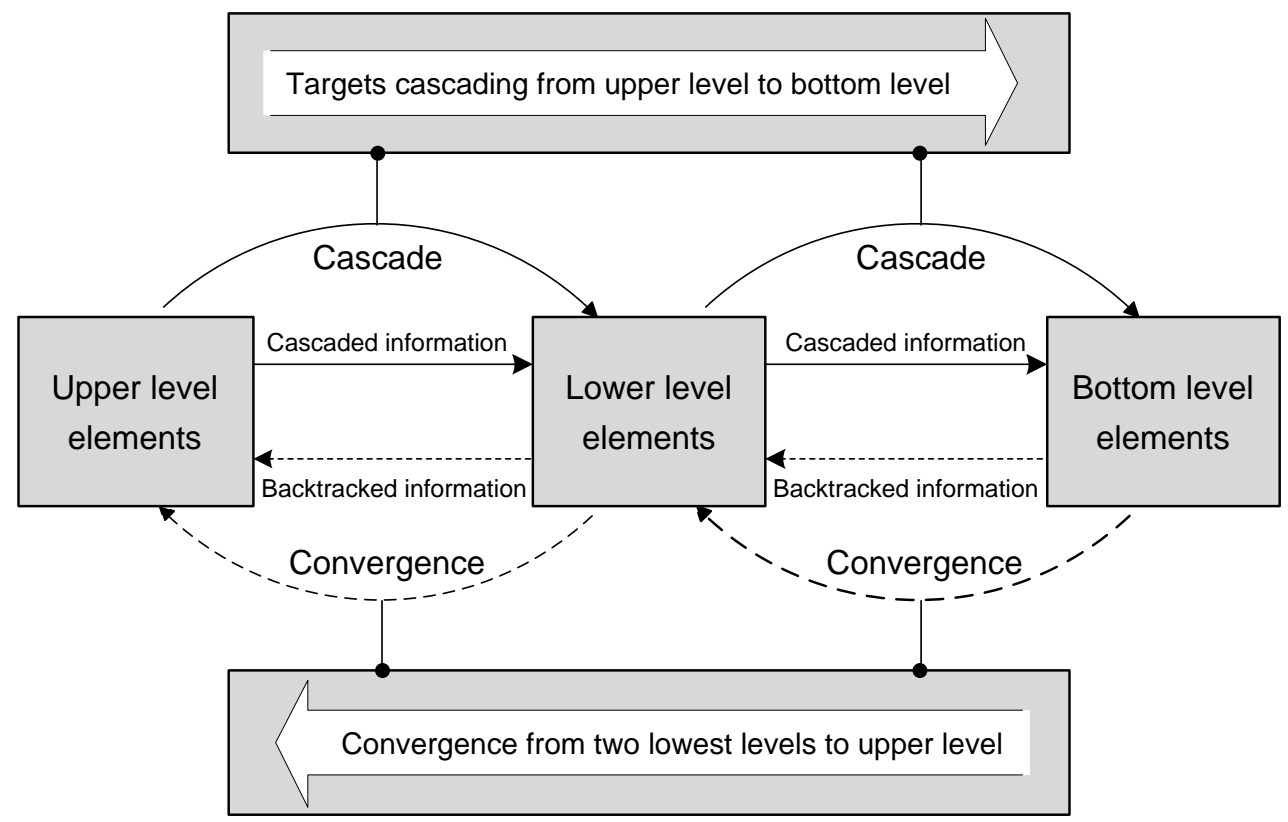

Fig. 6. The strategy of "lower levels converge first"

The convergence strategy for the whole ATC model mainly refers to the convergence sequence and converging criterion. Fig. 6 shows the convergence sequence, namely "lower levels converge first" that is adopted to implement the ATC model in this paper. Firstly, manufacturing targets are cascaded from the upper levels elements to the bottom level elements. Then, the elements in the two lowest levels are solved and coordinated until the convergence criterion is reached. This loop is expanded to include more and more upper levels until the top level. A balanced state is required when the coordination or consistency is achieved between one element and its child elements. Convergence criterion is used to judge whether the balance state has been reached. It can be presented as follows:

$$
\left|f_{I}\left(R_{k+1}, x_{k+1}\right)-f_{I}\left(R_{k}, x_{k}\right)\right| \leq \delta
$$

$f_{I}$ is the objective function of element $I . \quad k+1$ and $k$ denote the $(k+1)$ th and $k$ th iterations between element $I$ and its child elements. $x_{k}$ represents the vector of local variables of element $I . R_{k}$ represents the vector of the key links which are backtracked from the child elements. $\delta$ is the tolerance for $\left|f_{I}\left(R_{k+1}, x_{k+1}\right)-f_{I}\left(R_{k}, x_{k}\right)\right|$. It can be either a small absolute value or a 
small percentage of $f_{I}\left(R_{k}, x_{k}\right)$. When the equation (41) is satisfied, the balance state is reached between element $I$ and its child elements.

\subsection{Particle swarm optimization for each individual element}

In the ATC mechanism, there is no restriction to the optimization method for each individual element. Whether an optimization method is suitable for the individual element mainly depends on the application specifics. MSC is one kind of the resource allocation problem. By offering advantages of high efficiency, fast convergence and simple implementation, particle swarm optimization (PSO) was successfully applied to the resource allocation problems (Gong et al., 2012). Thus, in this paper, the PSO method is used to solve the optimization problems of these individual elements.

PSO is a swarm intelligence based metaheuristic algorithm proposed by Kennedy and Eberhart (1995) taking the inspiration from cooperation and communication of bird flocking or fish schooling (Alam et al., 2014). In PSO, each possible solution is represented as an individual particle, each of which is associated with two vectors, namely the velocity vector $v_{i}(t)$ and position $x_{i}(t)$, where $t$ stands for the iteration times in the evolutionary process. The velocity and position of each particle are initialized by random vectors within corresponding ranges. During the evolutionary process, the velocity and position of particle $i$ are updated as follows:

$$
\begin{gathered}
v_{i}(t+1)=w \cdot v_{i}(t)+\varphi_{1} \cdot \operatorname{rand}_{1} \cdot\left(p_{i}-x_{i}(t)\right)+\varphi_{2} \cdot \operatorname{rand}_{2} \cdot\left(p_{g}-x_{i}(t)\right) \\
x_{i}(t+1)=x_{i}(t)+v_{i}(t+1)
\end{gathered}
$$

Where $w$ denotes the inertia weight, $\varphi_{1}$ and $\varphi_{2}$ are the acceleration coefficients, rand $_{1}$ and rand $_{2}$ are two random numbers with uniform distribution in the range of $[0,1] . p_{i}$ is the position with the best fitness value found so far for the $i$ th particle (or the best history position for the $i$ th particle), and $p_{g}$ is the best position in the global swarm. The following procedure can be used for implementing the PSO to the individual elements in the ATC model by taking Element 2 for illustration.

Step 1: Initialize the random swarm by assigning a position to each particle. The assigned positions contain decision variables to be optimized, i.e. assign $x_{i}$ to the $i$ th particle and $x_{i}$ is presented as follows:

$$
x_{i}=\left(T T_{5}, T C_{5}, T E_{5}, T T_{6}, T C_{6}, T E_{6}, T T_{7}, T C_{7}, T E_{7}, \varepsilon_{2.1}, \ldots, \varepsilon_{2.9}\right)
$$

Step 2: Evaluate the fitness function for each particle. The fitness function of element 2 could be presented as follows:

$$
\text { Fit }_{2}\left(x_{i}\right)=f_{2}\left(x_{i}\right)+P e_{2}\left(x_{i}\right)
$$


The fitness function is composed of the objective function and penalty function. As said before, $f_{2}$ is the objective function of element 2. $P e_{2}$ is the penalty function to deal with the constraints in the formulation of element 2. $P e_{2}$ is presented as follows, where $\rho$ is the penalty coefficient.

$$
P e_{2}=\frac{1}{\rho} \cdot\left\{\begin{array}{l}
\sum_{k=5}^{7}\left\{\left(\max \left(\left(\left|T T_{k}-T T_{k}^{k}\right|-\varepsilon_{2 .(k-4)}\right), 0\right)\right)+\left(\max \left(\left(\left|T C_{k}-T C_{k}^{k}\right|-\varepsilon_{2 .(k-1)}\right), 0\right)\right)\right. \\
\left.+\left(\max \left(\left(\left|T E_{k}-T E_{k}^{k}\right|-\varepsilon_{2 .(k+2)}\right), 0\right)\right)\right\}
\end{array}\right\}
$$

Step 3: For the individual particle $i$, comparisons of the particle's fitness with its $p_{i}$ are conducted. If the value of $F_{2} t_{2}\left(x_{i}\right)$ is better than the value of $\operatorname{Fit}_{2}\left(p_{i}\right)$, then set the position of $x_{i}$ as $p_{i}$.

Step 4: Identify the particle whose best history position has the best fitness value among all the particles and set the best history position of this particle as $p_{g}$.

Step 5: Update the velocities and positions of all the particles according to equation (42) and equation (43).

Step 6: Repeat steps 2-5 until a stopping criterion is met (e.g. maximum iteration times or sufficient good fitness value).

\section{Case study and results analysis}

The fifth step is to analyze the optimization results. In this section, the optimization results are discussed from a case study.

As shown in Fig. 7, a typical engine case is presented. Generally, an engine is composed of several parts. For simplicity of understanding, three key parts (i.e. crankcase, EGR (Exhaust Gas Recirculation) passage and valve) are considered in this model. When the manufacturing task is submitted to the CMfg platform, all the manufacturing stages are represented as different manufacturing nodes in a three-level structure. Through analyzing the real-time manufacturing capabilities of manufacturing services in the service pool, the CMfg platform acquires all the available services for each manufacturing node. Crankcase, EGR passage and valve are respectively represented as element 2, 3 and 4. Meanwhile, elements 5-7 represent necessary manufacturing processes to produce crankcase, elements 8-11 represent necessary processes to produce EGR passage, elements 12-14 represent necessary processes to produce valve. Table 1 lists information of candidate services which comes from Guangdong Zhaoqing Automotive parts Association. About 31 candidate manufacturing services from five different service providers are considered in the case study. The information includes service style, manufacturing cost $(c)$, time $(t)$ and energy consumption $(e)$. Note that the data shown in Table 1 for each service is representative to keep the confidentiality of their key business. The values marked with "*” in the " $c$ " column respectively

represent different maintenance cost per unit time of manufacturing cells (i.e., $O_{2}^{1}, O_{3}^{1}, O_{3}^{2}$ and 
$O_{4}^{1}$ ). The combination of weighted manufacturing time, cost and energy consumption is taken as the optimization objective during the whole configuration process. Input parameters in this MSC problem mainly include the weighting coefficients $\left(w_{1}^{T}, w_{1}^{C}, w_{1}^{E}\right)$ and manufacturing targets $\left(t^{T}\right.$, $t^{C}, t^{E}$ ) for total time, total cost and total energy consumption. The weighting coefficients are respectively set as $0.25,0.25$, and 0.5 . The manufacturing targets are respectively set as zero.

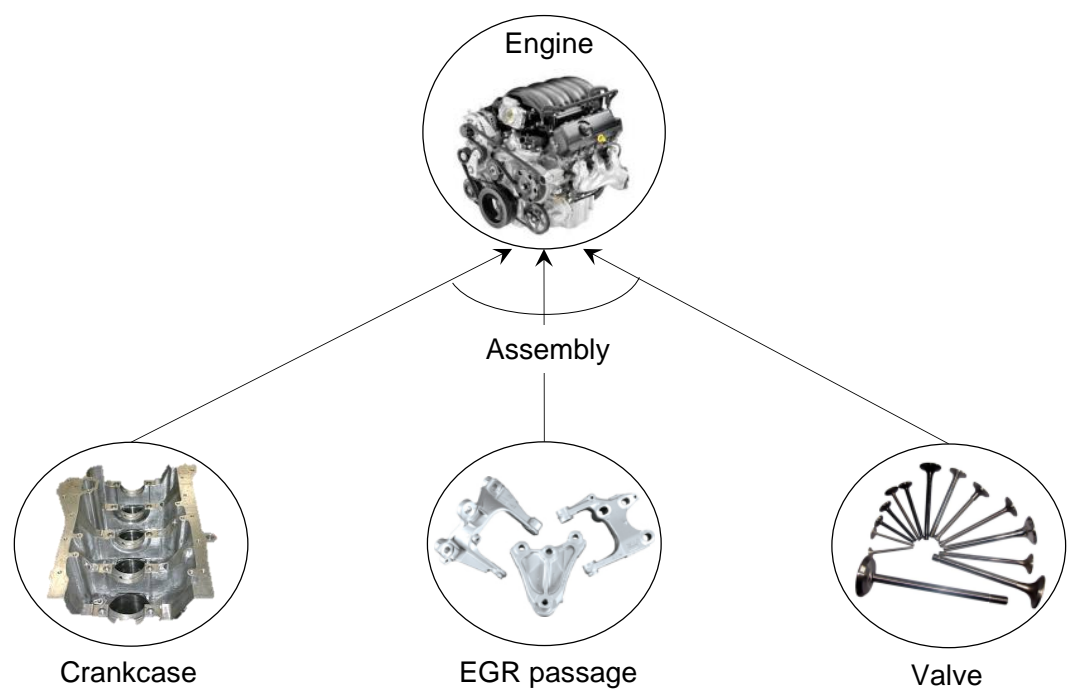

Fig. 7. Practical relevance of case model

Table 1 Information of all service options for each element

\begin{tabular}{|c|c|c|c|c|c|c|}
\hline \multicolumn{2}{|c|}{ Element } & \multicolumn{5}{|c|}{ Service information } \\
\hline Number & Belongs to & $\operatorname{Option}\left(O_{i}^{j}\right)$ & Service style & $c$ & $t$ & $e$ \\
\hline 1 & System level set & $O_{1}^{1}$ & System & 10 & 5 & 2 \\
\hline 2 & Cell level set & $\mathrm{O}_{2}^{1}$ & Manufacturing cell & $0.6^{*}$ & 0 & 1.2 \\
\hline \multirow[t]{2}{*}{3} & Cell level set & $O_{3}^{1}$ & Manufacturing cell & $0.5^{*}$ & 0 & 1.0 \\
\hline & & $O_{3}^{2}$ & Manufacturing cell & $0.7 *$ & 0 & 1.5 \\
\hline 4 & Cell level set & $O_{4}^{1}$ & Manufacturing cell & $0.5^{*}$ & 0 & 0.8 \\
\hline \multirow[t]{2}{*}{5} & Machine level set & $O_{5}^{1}$ & Machine & 5 & 10 & 2.5 \\
\hline & & $O_{5}^{2}$ & Machine & 6 & 5 & 2.0 \\
\hline \multirow[t]{2}{*}{6} & Machine level set & $O_{6}^{1}$ & Machine & 3 & 6 & 1.0 \\
\hline & & $O_{6}^{2}$ & Machine & 2 & 10 & 0.8 \\
\hline \multirow[t]{2}{*}{7} & Machine level set & $O_{7}^{1}$ & Machine & 4 & 4 & 1.5 \\
\hline & & $O_{7}^{2}$ & Machine & 5 & 3 & 1.6 \\
\hline
\end{tabular}




\begin{tabular}{|c|c|c|c|c|c|c|}
\hline \multirow[t]{4}{*}{8} & Machine level set & $O_{8}^{1}$ & Machine & 2 & 20 & 2.0 \\
\hline & & $O_{8}^{2}$ & Machine & 4 & 15 & 2.5 \\
\hline & & $O_{8}^{3}$ & Machine & 5 & 10 & 3.0 \\
\hline & & $O_{8}^{4}$ & Machine & 7 & 5 & 3.5 \\
\hline \multirow[t]{3}{*}{9} & Machine level set & $O_{9}^{1}$ & Machine & 6 & 16 & 1.5 \\
\hline & & $O_{9}^{2}$ & Machine & 8 & 9 & 2.0 \\
\hline & & $O_{9}^{3}$ & Machine & 10 & 4 & 2.5 \\
\hline \multirow[t]{4}{*}{10} & Machine level set & $O_{10}^{1}$ & Machine & 8 & 10 & 2.8 \\
\hline & & $O_{10}^{2}$ & Machine & 4 & 18 & 2.0 \\
\hline & & $O_{10}^{3}$ & Machine & 6 & 12 & 2.5 \\
\hline & & $O_{10}^{4}$ & Machine & 10 & 6 & 3.0 \\
\hline \multirow[t]{3}{*}{11} & Machine level set & $O_{11}^{1}$ & Machine & 6 & 7 & 2.5 \\
\hline & & $O_{11}^{2}$ & Machine & 3 & 14 & 1.5 \\
\hline & & $O_{11}^{3}$ & Machine & 5 & 10 & 2.0 \\
\hline \multirow[t]{2}{*}{12} & Machine level set & $O_{12}^{1}$ & Machine & 9 & 11 & 1.0 \\
\hline & & $O_{12}^{2}$ & Machine & 10 & 6 & 2.0 \\
\hline \multirow[t]{2}{*}{13} & Machine level set & $O_{13}^{1}$ & Machine & 5 & 8 & 1.2 \\
\hline & & $O_{13}^{2}$ & Machine & 6 & 4 & 1.8 \\
\hline \multirow[t]{2}{*}{14} & Machine level set & $O_{14}^{1}$ & Machine & 7 & 6 & 1.5 \\
\hline & & $O_{14}^{2}$ & Machine & 5 & 10 & 2.5 \\
\hline
\end{tabular}

\subsection{The effectiveness of ATC method in solving MSC problem}

To verify the effectiveness of ATC method in solving MSC problem, a PSO-based centralized optimization method was also tested in the same computing environment. The software MATLAB R2012b running on a PC at $2.80 \mathrm{GHz}$ with $6.00 \mathrm{~GB}$ RAM was adopted to obtain the optimization results. Table 2 contrasts the optimal manufacturing service configuration results obtained by ATC method and PSO-based centralized optimization method. The "value of objective function" listed in Table 2 refers to the value of $\left(w_{1}^{T} \cdot T T_{1}+w_{1}^{C} \cdot T C_{1}+w_{1}^{E} \cdot T E_{1}\right)$. As it can be seen, the ATC method achieves the same configuration results (i.e. service options, total manufacturing time, manufacturing cost, and energy consumption) as the PSO-based centralized optimization method.

The computation time of two methods is compared in two different strategies. In the first strategy, ATC method is implemented on a single PC without a parallel and distributed computing environment. Despite getting the same configuration results, ATC method spends much longer optimization time than the PSO-based centralized optimization method to obtain the optimal results. As shown in the last line of Table 2, ATC method takes 29.381 seconds to complete the whole optimization process and the PSO-based centralized optimization method uses 10.066 seconds. 
There are two reasons. Firstly, the complex key links are emerged among individual elements after the MSC problem being partitioned into a hierarchical ATC model. For example, there are nine lower level responses and nine corresponding tolerance variables in the decision model of element 2. The formulations of ATC elements become much more complex. That imposes a huge challenge on the computing efficiency of getting optimization results. Secondly, the ATC method requires a parallel and distributed computing environment. However, in this paper the whole optimization process of ATC method is finished in a single PC. In the second strategy, a parallel and distributed computing environment is constructed. According to the proposed hierarchical model, the entire problem solving process with ATC method is separated into 5 pieces. The first piece $P_{1}$ is to solve element 1 . Other pieces (i.e. $P_{2}, P_{3}, P_{4}$, and $P_{5}$ ) are to solve the elements with autonomous decision rights and their corresponding upstream elements. $P_{2}, P_{3}, P_{4}$, and $P_{5}$ are parallel pieces. Let $C T_{i}$ represent computation time of each piece, the whole computation time can be calculated as $C T_{1}+\max \left\{C T_{2}, C T_{3}, C T_{4}, C T_{5}\right\}$. After getting the optimal results, computation time for each piece is respectively $1.887 \mathrm{~s}, 5.663 \mathrm{~s}, 6.272 \mathrm{~s}, 4.401 \mathrm{~s}$, and $5.227 \mathrm{~s}$. The whole computation time is calculated as $8.159 \mathrm{~s}$. In this case, ATC method shows a better performance in a parallel and distributed computing environment compared to centralized method. The computing efficiency can be greatly improved when the ATC optimization process is executed by parallel and distributed computing.

Table 2 Optimization results of ATC method and PSO-based centralized optimization method

\begin{tabular}{|c|c|c|c|}
\hline \multicolumn{2}{|c|}{ Element } & \multirow{2}{*}{$\begin{array}{c}\text { Centralized optimization method } \\
\text { Service Option } \\
\end{array}$} & \multirow{2}{*}{$\begin{array}{c}\text { ATC method } \\
\text { Service Option }\end{array}$} \\
\hline Number & Belongs to & & \\
\hline 1 & System level set & $O_{1}^{1}$ & $O_{1}^{1}$ \\
\hline 2 & Cell level set & $\mathrm{O}_{2}^{1}$ & $\mathrm{O}_{2}^{1}$ \\
\hline 3 & Cell level set & $O_{3}^{2}$ & $O_{3}^{2}$ \\
\hline 4 & Cell level set & $O_{4}^{1}$ & $O_{4}^{1}$ \\
\hline 5 & Machine level set & $O_{5}^{2}$ & $O_{5}^{2}$ \\
\hline 6 & Machine level set & $O_{6}^{1}$ & $O_{6}^{1}$ \\
\hline 7 & Machine level set & $O_{7}^{2}$ & $O_{7}^{2}$ \\
\hline 8 & Machine level set & $O_{8}^{4}$ & $O_{8}^{4}$ \\
\hline 9 & Machine level set & $O_{9}^{3}$ & $O_{9}^{3}$ \\
\hline 10 & Machine level set & $O_{10}^{4}$ & $O_{10}^{4}$ \\
\hline 11 & Machine level set & $O_{11}^{3}$ & $O_{11}^{3}$ \\
\hline 12 & Machine level set & $O_{12}^{2}$ & $O_{12}^{2}$ \\
\hline
\end{tabular}




\begin{tabular}{lccc}
13 & Machine level set & $O_{13}^{2}$ & $O_{13}^{2}$ \\
14 & Machine level set & $O_{14}^{1}$ & $O_{14}^{1}$ \\
\hline Total manufacturing time & 30 & 30 \\
Total manufacturing cost & 112.9 & 112.9 \\
Total energy consumption & 191.6 & 191.6 \\
Value of Object Function & 131.525 & 131.525 \\
\hline Computation time & $10.066 \mathrm{~s}$ & $29.381 \mathrm{~s}(8.159 \mathrm{~s})$ \\
\hline
\end{tabular}

\subsection{Maintaining the autonomous decision right of manufacturing service}

As a decentralized optimization mechanism, the ATC method allows service providers to exercise autonomous decision rights during the whole optimization process. In this section, a hypothetical scenario is made to test whether the autonomous decision right of manufacturing service could be maintained by ATC method. Assume that the manufacturing cell service $\left(O_{2}^{1}\right)$ for element 2 plays a critical role in saving the manufacturing cost, and service provider of $O_{2}^{1}$ has the decision right to set its own manufacturing target. Total manufacturing cost $T C_{2}$ will be minimized. Therefore, a local target term $\min T C_{2}$ will be added into the objective function of element 2.

Table 3 shows the optimization results with and without the local target $\min T C_{2}$. As it can be seen, $T C_{2}$ is reduced from 22.4 to 22 after the local target being set. It means that the autonomous decision right of service provider can be achieved when the ATC method is adopted to solve the MSC problem. However, the total manufacturing time $T T_{2}$ and energy consumption $T E_{2}$ are increased with different values (i.e. $T T_{2}$ is increased from 14 to15, $T E_{2}$ has been increased from 37.6 to 40). It is because the decreased total manufacturing cost of element 2 brings extra manufacturing time to its upstream element (i.e. $T T_{7}$ is increased from 3 to 4 ). Meanwhile, $T T_{7}$ positively correlates with $T T_{2}$ and $T E_{2}$, which are shown by the analysis model of element 2. Thus the total manufacturing time and energy consumption of element 2 are respectively increased when the manufacturing time of element 7 is increased. 
Table 3 Optimization results of with and without local target

\begin{tabular}{|c|c|c|c|c|c|c|c|c|}
\hline \multirow{2}{*}{ Element } & \multicolumn{4}{|c|}{ Without local target } & \multicolumn{4}{|c|}{ With local target: $\min T C_{2}$} \\
\hline & Option & $T T_{i}$ & $T C_{i}$ & $T E_{i}$ & Option & $T T_{i}$ & $T C_{i}$ & $T E_{i}$ \\
\hline 1 & $O_{1}^{1}$ & 30 & 112.9 & 191.6 & $O_{1}^{1}$ & 30 & 112.5 & 194 \\
\hline 2 & $O_{2}^{1}$ & 14 & 22.4 & 37.6 & $\mathrm{O}_{2}^{1}$ & 15 & 22 & 40 \\
\hline 3 & $O_{3}^{2}$ & 30 & 49.5 & 103 & $O_{3}^{2}$ & 30 & 49.5 & 103 \\
\hline 4 & $O_{4}^{1}$ & 16 & 31 & 41 & $O_{4}^{1}$ & 16 & 31 & 41 \\
\hline 5 & $O_{5}^{2}$ & 5 & 6 & 10 & $O_{5}^{2}$ & 5 & 6 & 10 \\
\hline 6 & $O_{6}^{1}$ & 6 & 3 & 6 & $O_{6}^{1}$ & 6 & 3 & 6 \\
\hline 7 & $O_{7}^{2}$ & 3 & 5 & 4.8 & $O_{7}^{1}$ & 4 & 4 & 6 \\
\hline 8 & $O_{8}^{4}$ & 5 & 7 & 17.5 & $O_{8}^{4}$ & 5 & 7 & 17.5 \\
\hline 9 & $O_{9}^{3}$ & 4 & 10 & 10 & $O_{9}^{3}$ & 4 & 10 & 10 \\
\hline 10 & $O_{10}^{4}$ & 6 & 10 & 18 & $O_{10}^{4}$ & 6 & 10 & 18 \\
\hline 11 & $O_{11}^{3}$ & 10 & 5 & 20 & $O_{11}^{3}$ & 10 & 5 & 20 \\
\hline 12 & $O_{12}^{2}$ & 6 & 10 & 12 & $O_{12}^{2}$ & 6 & 10 & 12 \\
\hline 13 & $O_{13}^{2}$ & 4 & 6 & 7.2 & $O_{13}^{2}$ & 4 & 6 & 7.2 \\
\hline 14 & $O_{14}^{1}$ & 6 & 7 & 9 & $O_{14}^{1}$ & 6 & 7 & 9 \\
\hline
\end{tabular}

\section{Conclusions}

Cloud manufacturing (CMfg) is a service-oriented, high efficiency and low energy consumption manufacturing mode whose key technologies are widely paid attention by the academia and industry practitioners. Among these technologies, MSC plays an important role in facilitating the management and development of CMfg. This paper is considered as an attempt in introducing ATC methodology to deal with the MSC problem when a single manufacturing task is submitted to the CMfg platform.

This research brings four contributions. First is the introduction of a decentralized mechanism to cloud manufacturing management. For implementing cloud manufacturing, a decentralized strategy is more practical than traditional AIO (all-in-one) method. By using ATC in practice, users or administrators only need to adjust the number of subtasks or processes after manufacturing task submission. It is much more intelligent for decision making in cloud manufacturing without designing optimization parameters. Second is the identified five steps of applying ATC method to solve MSC problem in CMfg. (1) ATC modeling of MSC problem. Through the ATC modeling, MSC is converted into a hierarchical structure with three levels (i.e. system level, cell level, and machine level). Manufacturing stages, named ATC elements, can easily configure their own stages in a parallel way. (2) Identification of key links between related ATC elements, which provides the basis for the ATC optimization process. (3) Formulation of each individual ATC elements. This step embodies the decision models of individual elements with three major modules named objective function, analysis model and constraints. (4) ATC solutions for MSC problem. The overall 
convergence strategy and local optimization method are identified in this step. (5) Analysis of the optimization results. Third is the utilization of "OR" element to solve the MSC problem in which service providers own autonomous decision rights to configure their upstream manufacturing stages. During the whole ATC optimization process, OR element is employed to accomplish the selection of alternative manufacturing services according to its working logic and related evaluation criteria. The application of OR element enables the service providers to obtain autonomous decision rights during MSC process. Service providers are also encouraged to improve their performance to fulfill the cascaded manufacturing targets and increase the probability of obtaining manufacturing task. Fourth is the verified effectiveness of ATC method in solving the MSC problem. A case study was implemented by ATC method and centralized optimization method. ATC method can not only achieve the same optimization results as the centralized optimization method but also demonstrate promising features that centralized optimization method cannot offer. Because of its decentralized decision-making mechanism, ATC method allows individual manufacturing service providers to maintain their own decision models and choose their own local solution methods. Meanwhile, service providers are enabled to set their local targets to keep their flexibility and achieve sustainable competitive advantages in production process.

The future research may follow several aspects. Firstly, how to tackle the MSC problem when the same manufacturing services are shared by two or more downstream manufacturing stages? It is worth making some investigations to test whether this type of MSC problem can be converted into a traditional ATC model by some ancillary methods. Secondly, how to deal with the MSC problem when a large batch of manufacturing tasks are submitted to the CMfg platform? It is much more complex than the investigated problem in this paper. Thirdly, how to develop new strategies to decompose and represent the MSC problem into an ATC model? The number of manufacturing nodes and key links in the ATC model can affect the complexity and computational efficiency. Future studies should be conducted to find the right decomposing strategy to minimize the complexity and computational efficiency for the MSC problem. Fourthly, how to encapsulate the ATC method into a service and publish it on the CMfg platform? It will unfold more potential of ATC method in solving MSC problem. Finally, analysis of legal aspects, quality management, and logistics will be important in implementing sustainable cloud manufacturing system. For example, as one part of legal aspects, legal compliance can ensure the compliance through all phases of manufacturing system development (Wadhwa, 2015). Quality management aims to manage manufacturing businesses to meet or exceed customers' expectations. It is considered to be suitable as a support for the integration of sustainability considerations in areas such as manufacturing system management (Siva et al., 2016). In addition, sensitivity and robustness analysis of solutions should be another future work.

\section{Acknowledgements}

The authors would like to acknowledge the financial supports of National Science Foundation of China (51675441, 51475095) and the 111 Project Grant (B13044). This research is also supported in part by the Mistra REES (Resource Efficient and Effective Solutions) program funded by MISTRA, the Swedish Foundation for Strategic Environmental Research (No. 2014/16) and the Circularis (Circular Economy through Innovating Design) project funded by VINNOVA, Sweden's Innovation Agency (No. 2016-03267). Special thanks go to Guangdong Zhaoqing Automotive Parts Industry Association. The authors wish to express their appreciation to the anonymous reviewers for their 
valuable comments and suggestions to improve this paper.

\section{References}

Alam, S., Dobbie, G., Koh, Y.S., Riddle, P., Ur Rehman, S., 2014. Research on particle swarm optimization based clustering: A systematic review of literature and techniques. Swarm Evol. Comput. 17, 1-13. doi:10.1016/j.swevo.2014.02.001

Buckholtz, B., Ragai, I., Wang, L., 2015. Cloud Manufacturing: Current Trends and Future Implementations. J. Manuf. Sci. Eng. 137, 40902_1-40902_9. doi:10.1115/1.4030009

Cao, Y., Wang, S., Kang, L., Gao, Y., 2016. A TQCS-based service selection and scheduling strategy in cloud manufacturing. Int. J. Adv. Manuf. Technol. 82, 235-251. doi:10.1007/s00170-015-7350-5

Cheng, Y., Tao, F., Liu, Y., Zhao, D., Zhang, L., Xu, L., 2013. Energy-aware resource service scheduling based on utility evaluation in cloud manufacturing system. Proc. Inst. Mech. Eng. Part B-Journal Eng. Manuf. 227, 1901-1915. doi:10.1177/0954405413492966

Elkhorchani, H., Grayaa, K., 2016. Novel home energy management system using wireless communication technologies for carbon emission reduction within a smart grid. J. Clean. Prod. 135, 950-962. doi:http://dx.doi.org/10.1016/j.jclepro.2016.06.179

Gong, Y.J., Zhang, J., Chung, H.S.H., Chen, W.N., Zhan, Z.H., Li, Y., Shi, Y.H., 2012. An Efficient Resource Allocation Scheme Using Particle Swarm Optimization. Ieee Trans. Evol. Comput. 16, 801-816. doi:10.1109/tevc.2012.2185052

Huang, B., Li, C., Tao, F., 2014. A chaos control optimal algorithm for QoS-based service composition selection in cloud manufacturing system. Enterp. Inf. Syst. 8, 445-463. doi:10.1080/17517575.2013.792396

Huang, G.Q., Qu, T., 2008. Extending analytical target cascading for optimal configuration of supply chains with alternative autonomous suppliers. Int. J. Prod. Econ. 115, 39-54. doi:10.1016/j.ijpe.2008.04.008

Jiang, P., Wang, J., Zhou, Q., Zhang, X., 2015. An enhanced analytical target cascading and Kriging model combined approach for multidisciplinary design optimization. Math. Probl. Eng. 2015, 1-11. doi: $10.1155 / 2015 / 685958$

Kang, N., Kokkolaras, M.., Papalambros, P.Y.., Yoo, S.., Na, W.., Park, J.., Featherman, D.., 2014a. Optimal design of commercial vehicle systems using analytical target cascading. Struct. Multidiscip. Optim. 50, 1103-1114. doi:10.1007/s00158-014-1097-8

Kang, N., Kokkolaras, M., Papalambros, P.Y., 2014b. Solving multiobjective optimization problems using quasi-separable MDO formulations and analytical target cascading. Struct. Multidiscip. Optim. 50, 849-859. doi:10.1007/s00158-014-1144-5

Kennedy, J., Eberhart, R., 1995. Particle swarm optimization. Neural Networks, 1995. Proceedings., IEEE Int. Conf. 4, 1942-1948. doi:10.1109/ICNN.1995.488968

Kim, H.M., Michelena, N.F., Papalambros, P.Y., Jiang, T., 2003a. Target Cascading in Optimal System Design. J. Mech. Des. 125, 474-480. doi:10.1115/1.1582501 
Kim, H.M., Rideout, D.G., Papalambros, P.Y., Stein, J.L., 2003b. Analytical Target Cascading in Automotive Vehicle Design. J. Mech. Des. 125, 481-489. doi:10.1115/1.1586308

Laili, Y., Tao, F., Zhang, L., Sarker, B.R., 2012. A study of optimal allocation of computing resources in cloud manufacturing systems. Int. J. Adv. Manuf. Technol. 63, 671-690. doi:10.1007/s00170-012-3939-0

Lartigau, J., Xu, X., Nie, L., Zhan, D., 2015. Cloud manufacturing service composition based on QoS with geo-perspective transportation using an improved Artificial Bee Colony optimisation algorithm. Int. J. Prod. Res. 53, 4380-4404. doi:10.1080/00207543.2015.1005765

Li, C.Z., Hong, J., Xue, F., Shen, G.Q., Xu, X., Mok, M.K., 2015. Schedule risks in prefabrication housing production in Hong Kong: A social network analysis. J. Clean. Prod. 134, 482-494. doi:10.1016/j.jclepro.2016.02.123

Li, Z., Kokkolaras, M., Papalambros, P., Hu, S.J., 2008. Product and Process Tolerance Allocation in Multistation Compliant Assembly Using Analytical Target Cascading. J. Mech. Des. 130, 091701_1-091701_9.doi:10.1115/1.2943296

Liu, H., Chen, W., Kokkolaras, M., Papalambros, P.Y., Kim, H.M., 2006. Probabilistic Analytical Target Cascading: A Moment Matching Formulation for Multilevel Optimization Under Uncertainty. J. Mech. Des. 128, 991-1000. doi:10.1115/1.2205870

Liu, W., Liu, B., Sun, D., Li, Y., Ma, G., 2013. Study on multi-task oriented services composition and optimisation with the "Multi-Composition for Each Task" pattern in cloud manufacturing systems. Int. J. Comput. Integr. Manuf. 26, 786-805. doi:10.1080/0951192X.2013.766939

Liu, Y., 2013. Sustainable competitive advantage in turbulent business environments. Int. J. Prod. Res. 51, 2821-2841. doi:10.1080/00207543.2012.720392

Liu, Y., Liang, L., 2015. Evaluating and developing resource-based operations strategy for competitive advantage: an exploratory study of Finnish high-tech manufacturing industries. Int. J. Prod. Res. 53, 1019-1037. doi:10.1080/00207543.2014.932936

Michalek, J.J., Feinberg, F.M., Papalambros, P.Y., 2005. Linking marketing and engineering product design decisions via analytical target cascading. J. Prod. Innov. Manag. 22, 42-62. doi:10.1111/j.0737-6782.2005.00102.x

Michalek, J.J., Papalambros, P.Y., 2005. An Efficient Weighting Update Method to Achieve Acceptable Consistency Deviation in Analytical Target Cascading. J. Mech. Des. 127, 206-214. doi:10.1115/1.1830046

Qu, T., Huang, G.Q., Cung, V.-D., Mangione, F., 2010a. Optimal configuration of assembly supply chains using analytical target cascading. Int. J. Prod. Res. 48, 6883-6907. doi:10.1080/00207540903307631

Qu, T., Huang, G.Q., Zhang, Y., Dai, Q.Y., 2010b. A generic analytical target cascading optimization system for decentralized supply chain configuration over supply chain grid. Int. J. Prod. Econ. 127, 262-277. doi:10.1016/j.ijpe.2009.08.008

Qu, T., Lei, S.P., Wang, Z.Z., Nie, D.X., Chen, X., Huang, G.Q., 2016. IoT-based real-time production logistics synchronization system under smart cloud manufacturing. Int. J. Adv. Manuf. Technol. 84, 
147-164. doi:10.1007/s00170-015-7220-1

Rauch, E., Dallasega, P., Matt, D.T., 2016. Sustainable production in emerging markets through Distributed Manufacturing Systems (DMS). J. Clean. Prod. 135, 127-138. doi:10.1016/j.jclepro.2016.06.106

Ren, L., Zhang, L., Zhao, C., Chai, X., 2013. Cloud Manufacturing Platform: Operating Paradigm, Functional Requirements, and Architecture Design. Proc. ASME 2013 Int. Manuf. Sci. Eng. Conf. 2, 1-10. doi:10.1115/MSEC2013-1185

Shrouf, F., Miragliotta, G., 2015. Energy management based on Internet of Things: practices and framework for adoption in production management. J. Clean. Prod. 100, 235-246. doi:10.1016/j.jclepro.2015.03.055

Siva, V., Gremyr, I., Bergquist, B., Garvare, R., Zobel, T., Isaksson, R., 2016. The support of Quality Management to sustainable development : a literature review. J. Clean. Prod. 138, 148-157. doi:10.1016/j.jclepro.2016.01.020

Sun, H., Liu, Y., Sakao, T., Wang, Z., 2016. Configuring use-oriented aero-engine overhaul service with multi-objective optimization for environmental sustainability. J. Clean. Prod. In Press, 1-13. doi:10.1016/j.jclepro.2016.12.022

Tao, F., Cheng, Y., Xu, L. Da, Zhang, L., Li, B.H., 2014a. CCIoT-CMfg: Cloud computing and internet of things-based cloud manufacturing service system. IEEE Trans. Ind. Informatics 10, 1435-1442. doi:10.1109/TII.2014.2306383

Tao, F., Zuo, Y., Xu, L. Da, Zhang, L., 2014b. IoT-Based intelligent perception and access of manufacturing resource toward cloud manufacturing. IEEE Trans. Ind. Informatics 10, 1547-1557. doi:10.1109/TII.2014.2306397

Tosserams, S., Kokkolaras, M., Etman, L.F.P., Rooda, J.E., 2008. Extension of Analytical Target Cascading using Augmented Lagrangian Coordination for Multidisciplinary Design Optimization. Proc. 12th AIAA/ISSMO Multidiscip. Anal. Optim. 5843, 1-9. doi:doi: 10.2514/6.2008-5843

Wadhwa, R.S., 2015. Towards addressing Legal Compliance in Manufacturing Automation Systems. Int. J. Comput. Sci. Issues 12, 129-133.

Xiang, F., Hu, Y., Yu, Y., Wu, H., 2014. QoS and energy consumption aware service composition and optimal-selection based on Pareto group leader algorithm in cloud manufacturing system. Cent. Eur. J. Oper. Res. 22, 663-685. doi:10.1007/s10100-013-0293-8

Xiang, F., Jiang, G.Z., Xu, L.L., Wang, N.X., 2016. The case-library method for service composition and optimal selection of big manufacturing data in cloud manufacturing system. Int. J. Adv. Manuf. Technol. 84, 59-70. doi:10.1007/s00170-015-7813-8

Xu, W., Tian, S., Liu, Q., Xie, Y., Zhou, Z., Pham, D.T., 2016. An improved discrete bees algorithm for correlation-aware service aggregation optimization in cloud manufacturing. Int. J. Adv. Manuf. Technol. 84, 17-28. doi:10.1007/s00170-015-7738-2

Xu, X., 2012. From cloud computing to cloud manufacturing. Robot. Comput. Integr. Manuf. 28, 75-86. doi:10.1016/j.rcim.2011.07.002

Zhang, Y., Qian, C., Lv, J., Liu, Y., 2016a. Agent and cyber-physical system based self-organizing and 
self-adaptive intelligent shopfloor. IEEE Trans. Ind. Informatics In press, 1-10.

doi:10.1109/TII.2016.2618892

Zhang, Y., Ren, S., Liu, Y., Si, S., 2017a. A big data analytics architecture for cleaner manufacturing and maintenance processes of complex products. J. Clean. Prod. 142, 626-641.

doi:10.1016/j.jclepro.2016.07.123

Zhang, Y., Wang, J., Liu, S., Qian, C., 2017b. Game Theory Based Real-Time Shop Floor Scheduling Strategy and Method for Cloud Manufacturing. Int. J. Intell. Syst. 32, 437-463. doi:10.1002/int.21868

Zhang, Y., Wang, W., Wu, N., Qian, C., 2016b. IoT-Enabled Real-Time Production Performance Analysis and Exception Diagnosis Model. IEEE Trans. Autom. Sci. Eng. 13, 1318-1332. doi:10.1109/TASE.2015.2497800

Zhang, Y., Zhang, G., Liu, Y., Hu, D., 2015. Research on services encapsulation and virtualization access model of machine for cloud manufacturing. J. Intell. Manuf. In press, 1-15.

doi:10.1007/s10845-015-1064-2

Zheng, H., Feng, Y., Tan, J., 2016. A fuzzy QoS-aware resource service selection considering design preference in cloud manufacturing system. Int. J. Adv. Manuf. Technol. 84, 371-379.

doi:10.1007/s00170-016-8417-7 